\title{
Epidemiology of Scars and Their Consequences: Burn Scars
}

Margriet E. van Baar

\section{Contents}

\subsection{Burn Injuries and Their Treatment - 38}

5.1.1 Burn Care - 38

5.1.2 Acute Phase -38

5.2 Prevalence of Burn Scars and Their Consequences - 39

5.2.1 Definition of Scars - 39

5.2.2 Prevalence of Hypertrophic Scarring - 39

5.2.3 Prevalence of Contractures - 40

5.2.4 Scar Quality Assessment - 40

5.2.5 Prevalence of Reconstructive Surgery - 40

5.2.6 Maturation Pattern - 40

5.3 Factors Predicting Scar Outcome After Burns - 41

5.3.1 Patient Characteristics - 41

5.3.2 Injury and Treatment Characteristics - 41

5.3.3 Patient, Injury and Treatment Characteristics Combined - 42

5.4 Clinical Relevance - 42

5.5 Conclusion -42

References - 43 
Background

This chapter focuses on the epidemiology of burn scars and their consequences. Burn scars are the result of a pathological healing process in specific burn injuries.

The occurrence of burn injuries and resulting scars and their consequences will be discussed. This includes scar contractures and reconstructive surgery. Next, the predisposing factors for the occurrence of burn scars will be addresses. The chapter ends with the clinical implications.

\subsection{Burn Injuries and Their Treatment}

Burn injuries are a global public health problem. According to estimates of the WHO, burn injuries cause 180,000 deaths every year. The majority of these deaths occur in low- and middle-income countries. Two-third of these deaths occur in the WHO regions of Africa and Southeast Asia.

The nonfatal burns are a leading cause of morbidity, including long hospitalizations, disability and problems in returning to normal life. In the United States annually, 486,000 burn injuries receive medical treatment. In addition, 40,000 hospitalizations are related to burn injuries.

National burn injury incidence data are scarce. In Europe, incidence rates of hospitalized burn injuries are reported between 2 and 29 per 100,000 inhabitants; in Australia, 36 per 100,000 has been reported.

Reviews on burn injury incidence data describe a decline in the incidence rates of burn-related hospital admissions in both low- and middle-income countries. In high-income countries, a similar trend is observed.

\subsubsection{Burn Care}

In the past decades, there has been a shift in focus from mortality to morbidity in burn care in high-income countries. Recently, the European Burns Association stated in its guidelines that "the main goal of burn care is to ensure optimum resuscitation in the emergency period and then to reach re-epithelialization of injured or destroyed skin either by support of spontaneous healing or by surgical necrectomy and grafting with split thickness skin graft. Subsequent treatment is to ensure the optimum post burn quality of life". This goal only includes the minimum surgical procedure. In nowadays burn care, other surgical techniques including skin substitutes, full skin transplantations and ReCell are also applied.

In high-income countries, a trend is described towards a growing specialized burn care. In the Netherlands, the majority of these hospital admissions are seen in specialized burn centres. This is in line with the trend towards further specialization of health care in general and also because of limited travel distances in our country. In addition, it has been suggested that many patients are transferred towards tertiary care facilities because of a lack of basic skills in the assessment and care of burn wounds at community and rural hospitals.

\subsubsection{Acute Phase}

The acute treatment of burn injuries is guided by their severity, indicated by the burn size and the burn depth. Burn size is represented by the percentage of the total body surface area burned (TBSA). The burn depth is related to the dermal layers which are affected by the burn injury. Accurate diagnosis of both burn size and burn depth is crucial to start appropriate treatment at the correct level of care required. It guides the initiation of fluid resuscitation, acute escharotomy in case of circumferential deep burns and the decision for conservative or surgical wound treatment. Burn size also has an important prognostic value in predicting burn-related mortality. Both burn size and burn depth are of prognostic value for eventual burn scar pathology.

The most widely used classification for burn injuries divides burns into three categories of increasing depth: superficial partial-thickness, deep partial-thickness and full-thickness burns. Superficial partial-thickness burns involve only the epidermal layer and the superficial part of the dermis. In deep partial-thickness burns, the epidermis and the majority of the dermis are destroyed, with damage to deeper skin structures such as blood vessels, nerves and hair follicles. In full-thickness burns, all layers of the skin are destroyed, and there may also be damage to subdermal structures, such as the muscle, cartilage or bone. In these wounds, no viable epidermal appendages remain in the bottom of the wound, which makes spontaneous healing from the wound bed impossible.

In superficial partial-thickness burns, conservative treatment will be chosen to support spontaneous wound healing, which is expected within 10-14 days. In deep partial-thickness burns, surgical treatment is sometimes necessary to reach wound closure. In full-thickness burns, surgical wound closure will be necessary in the majority of wounds, except in the very small-sized ones. 
Other important parts of burn care include oedema management, early mobilization and scar management.

Treatment protocols vary between burn centres, including wound dressings, early mobilization and timing of surgery. According to Dokter et al., in the Netherlands, $45 \%$ of all burn injury patients admitted to burn centres receive surgery approximately 15 days post burn. In burn centres in Australia and New Zealand, a similar proportion is operated upon $(47.2 \%)$ in general within 1 week post burn (mean 6 days post burn).

\subsection{Prevalence of Burn Scars and Their Consequences}

\subsubsection{Definition of Scars}

The consequences of burns can be severe, affecting multiple domains. The focus in this chapter is on the prevalence of pathological physical scar formation and its consequences.

Burn scars can cause aesthetic and functional problems, resulting in limitations in activities of daily living, the return to normal social roles and impacted quality of life. The psychological consequences will be further elucidated elsewhere in this book.

Early studies on pathological scarring in burn wounds have distinguished between hypertrophic scars and contractures (Magliacani (1997) in [1]).

Hypertrophic scars are a dominant type of pathological scar formation after burns. Nowadays, hypertrophic scarring is described as "the greatest unmet challenge after burn injury."

Hypertrophic scars are generally elevated, firm and erythematous. They can also tend to be pruritic and tender. By definition, they are limited to the site of the original wound and grow in size by pushing out the scar margins. Hypertrophic scarring is known to decrease with time.

Next to hypertrophic scars, contractures are another important type of pathological scar formation after burns. A contracture is defined as the pathological result of excessive scarring and ongoing scar contraction that results in loss of range of motion (ROM) over joint areas. Scar contractures may limit daily functioning and as a result affect health-related quality of life after burns.

Modern scar assessment addresses a broader range of scar features from both the patients' perspective and the perspective of the health-care professional. Scar quality from a patient perspective depends on the pres- ence or absence of several visual, tactile and sensational features [2]. For research purposes, an ideal scar evaluation protocol should include both subjective and objective measurements. These different perspectives will result in different scar assessment tools and different scar outcomes. Results indicate the level of scar morbidity and not the mere presence or absence of scar pathology.

Examples of frequently used scar assessment scales are the Vancouver Scar Scale and the Patient and Observer Scar Assessment Scale (POSAS; see $>$ www. posas.org).

A final approximation of burn scarring is the occurrence of reconstructive surgery after burns. The need for reconstructive surgery after burn injuries can be viewed upon as an indicator for problematic scar development, requiring a surgical intervention to relieve scar morbidity.

\subsubsection{Prevalence of Hypertrophic Scarring}

The estimates of prevalence of scarring vary widely. Prevalences of hypertrophic scarring between $8 \%$ and $67 \%$ are reported [3]. These data are all related to burn centre populations in high-income countries.

One of the first reports was written by Deitch et al. in 1983, based on a combined retrospective and prospective study in a mixed population of children and adults. They reported hypertrophic scars after conservative wound healing in $30 \%$ of their patients with a dark skin and in $15 \%$ of their Caucasian/White patients at 9 to 18 months post burn. Hypertrophic scars were documented in case of increased thickness or elevation of the burn wound; changes in colour or pigmentation alone were not classified as hypertrophy.

Two decades later, Bombardo reported a prevalence of $67 \%$ of hypertrophic scars in a dominantly Caucasian sample of patients after major burns. Again, data were based on retrospective chart analysis in patients after major burns, examining the mentioning of the word hypertrophic in the charts. No description was provided on the timing of the scar assessments.

More recently, estimates of prevalence of hypertrophic scars are derived on assessments using validated instruments. The Vancouver Scar Scale is traditionally a well-known scale to evaluate burn scars. In 2017, Wallace et al. assessed the prevalence of hypertrophic scars, using the Modified Vancouver Scar Scale. Raised hypertrophic scarring over $1 \mathrm{~mm}$ was reported in $7.8 \%$ of the patients treated in a Western Australian burns unit up to 1 year post burn [4]. 


\subsubsection{Prevalence of Contractures}

Although contractures are a dominant feature after burn injuries, data on prevalence of burn scar contractures are scarce. In a recent review by Oosterwijk [5] et al., only 10 relevant studies were available for inclusion. The prevalence at discharge was $38-54 \%$ and decreased with an increasing time post burns.

The included studies indicated an elevated risk for contractures in deep or surgically treated burn injuries, in females and children. In addition, contractures seemed to occur more frequently in the upper extremity joints, specifically in the shoulder and elbows.

Assessment of scar contractures was based on range of motion measurement in six studies; in four studies, the method of scar contracture assessment was not described. In addition, study design, study period, study population and timing of scar contracture assessment varied largely. Consequently, the authors concluded that "prevalence of scar contractures after burn is insufficiently reported and varies considerably between studies." This impedes the analysis of determinants of scar contractures and possible interventions [5].

Recently, Schouten et al. assessed scar contractures in a prospective cohort study using passive ROM assessments up to 12 months. Scar contractures one year post burn were only found in operated burned joints. Scar contractures at discharge in non-operated joints all returned back to normal in 6 to 9 months. Again, scar contractures were more often seen in the upper part of the body, especially in the shoulder [6].

\subsubsection{Scar Quality Assessment}

In contrast to the assessment of scar hypertrophy or scar contractures, scar quality assessment addresses a broader assessment of scar-related characteristics, including colour, thickness, relief, pliability, pain and pruritus. The Patient Scar Assessment Scale and the Patient and Observer Scar Assessment Scale (POSAS), for example, consist of the two six-item numeric scales reflecting the patient's perspective and the observer's perspective. This is in contrast to another frequently used scale, the Vancouver Scar Scale, which reflects the observer perspective only and has some methodological difficulties, especially concerning the item "pigmentation."

The POSAS was introduced in 2004. Both the selfreported mean patient POSAS and the observer POSAS are based on scores on six individual scar characteristics. These scar characteristics are scored on a numerical 10-point scale, in which 1 represents a scar comparable with "normal skin" whereas 10 represents the "worst scar imaginable."

Using the POSAS, substantial scar morbidity is reported, even several years after the burn injury. In patients after mild to intermediate burn injuries, mean self-reported patient POSAS scores varied between 1.8 and 2.9 more than 2 years post [7]. In a similar sample 5 years post burn, these patient POSAS scores were even higher, reflecting more scar morbidity. The mean patient POSAS was 3.0, and 7\% had a POSAS score above 4 (on a scale from 1 to 10) on all six scar characteristics.

In patients after severe burns (with a mean TBSA of $24.0 \%$ ), scar morbidity was higher, with a patient POSAS of 4.5 (SD 2.0) [8].

\subsubsection{Prevalence of Reconstructive Surgery}

The need for reconstructive surgery after burn injuries can be viewed upon as an indicator for problematic scar development, requiring a surgical intervention to relieve scar morbidity. Of course, the possibilities to perform reconstructive surgery will influence the occurrence of reconstructive surgery.

Recent studies in patients after a burn centre admission in the Netherlands showed that $13.0 \%$ of these patients received reconstructive surgery in a ten-year follow-up period after burn injuries. In patients with head and neck burns, the prevalence of reconstructive surgery was $8.9 \%$ [9]. Vlies et al. reported a prevalence of $15 \%$ in patients after hand burns. These prevalences are lower than the one reported in the early 1990s by Prasad et al. $(19.9 \%)$. This can probably be related not only to improvements in acute burn care between the 1970-1985 and 1990 but also to the more frequent use of non-surgical therapies in the rehabilitation phase, like silicones and laser therapy. Another explanation for these differences may be the higher burn size in the study of Prasad with a mean TBSA of $16.4 \%$ and $5.8 \%$ full-thickness burns, compared to our sample (9.8 and 3.4\%). Also follow-up time differed; patients in the study of Prasad were followed for a variable period of 5 to 20 years.

Another Dutch study by Hoogewerf et al. in patients after head and neck burns described a prevalence of $5.3 \%$ reconstructive surgery. This prevalence is lower than the one found in the 10-year follow-up period, probably because of the shorter follow-up period of 2-7 years post burn.

\subsubsection{Maturation Pattern}

In general, hypertrophic scars develop in the first months after the burn injury. Then, scar hypertrophy is described 


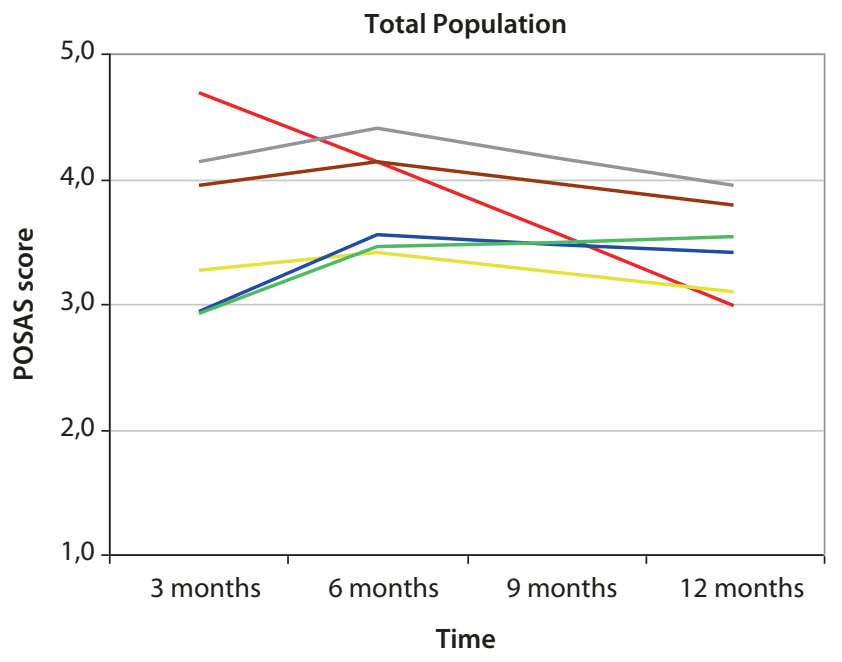

$\begin{array}{ll}- \text { Vascularisation } & - \text { Pliability } \\ \text { Pigmentation } & \text { Relief } \\ \text { Thickness } & - \text { Overall impression }\end{array}$

- Fig. 5.1 Maturation pattern after burn injuries, assessed with observer scales of POSAS (Van der Wal et al., personal communication; see also Wound Repair Regen. 2012;20:676-87)

to increase up to 6 months. At 12 months, there is a tendency to regress, according to several studies [2].

Individual scar characteristics have an individual maturation pattern. Vascularization shows a continuous decrease from 3 months post burn onwards, and relief and thickness show increased scar morbidity during at least the first year post injury (• Fig. 5.1).

\subsection{Factors Predicting Scar Outcome After Burns}

Several studies investigated the predictors of pathological scar formation after burn injuries.

Some groups of predictors can be distinguished, including patient, injury and treatment characteristics.

\subsubsection{Patient Characteristics}

A few patient characteristics are recognized for their predictive value in pathological scar formation.

Sex: The relation between sex and scar quality is ambiguous.

There are several studies reporting a poorer scar quality in females. In Dutch studies using the Patient and Observer Scar Assessment Scale (POSAS), a poorer scar quality was reported in females two and five years after burns. Observers reported a poorer scar quality in female participants two years post burn, but the females themselves reported similar scar quality, compared to their male counterparts. However, after taking skin type and surgical treatment into account, scar quality was similar [7].

In patients five years after burns, female participants reported a similar overall patient POSAS score but a poorer overall scar opinion, compared to men. This sex differences remained, also after controlling for other relevant factors including length of hospital stay [8].

In an Australian study predicting raised scars $(<1 \mathrm{~mm})$ in adults, based on the Modified Vancouver Scar Scale, females had an increased risk of raised scars, also after taking into account other relevant factors [4]. In a similar study in children, no gender differences were found.

However, in earlier studies, using a variety of scar assessments, this gender difference was not observed [1].

Age: An older age has been shown to reduce the risk for pathological scarring in several studies. The risk for raised scars was reduced in patients over 45 years of age, compared to patients 30 years of age and under [2]. In a paediatric study, the youngest children ( $0-5$ years) were at higher risk to develop raised scars [10].

Skin type: Skin type is a well-known factor influencing the scar formation and scar quality. In one of the first studies on prediction of scar quality from Deitch in 1983, scar hypertrophy was more often reported in patients with a darker skin. In recent studies, skin type is often classified using the Fitzpatrick skin type test. This score classifies skin types in six categories, from type I (palest) to type VI (deeply pigmented dark brown to darkest brown). Having skin types V or VI was associated with a poorer scar quality, based on both the patient and observer POSAS [7]. In addition, darker skin (skin types IV-VI) has an increased risk on raised scars [4]. In children, this association was not reported [10].

\subsubsection{Injury and Treatment Characteristics}

Several injury and treatment characteristics have been documented to influence scar formation and scar quality.

These include several burn severity indicators, including increased burn size (percentage of total body surface area burned (TBSA)), high \%TBSA full thickness, wound complications and a longer time to wound healing.

In addition, several treatment characteristics have been described to be related to poor scar quality, including a prolonged hospital stay, type of surgery (none, ReCell, skin grafting, dermal substitute), multi- 
ple surgeries, repeated surgery in the same wound, reconstructive surgery and artificial ventilation $[4,10]$. Again, all these treatment features are related to the severity of the burn injuries, complexity of wound healing and possible complications. Length of stay in these analyses is the representation or proxy for the severity of the burn injuries and the complexity of the burn care process including wound healing and possible complications.

\subsubsection{Patient, Injury and Treatment Characteristics Combined}

To identify the independent predictors of poor scar outcome, the potential predictors have to be taken into account together in one analysis, which requires adequate numbers to reach significant power of the analysis. Some examples of such studies are summarized here.

Raised scars in children up to 16 years of age, one year post burn, could be predicted by greater burn size (\%TBSA), a prolonged healing time (over 14 days) and multiple surgical procedures. Patient characteristics did not contribute to this prediction of scars after burn injuries in children [10].

Raised scars in adults one year post burn could be predicted again by increasing burn size (\%TBSA) and by the type of surgical intervention (as a proxy for burn depth), wound complications and prolonged hospital stay. In addition, patient predictors were found, being a young age $(<30)$, the female gender and darker skin were for raised scars in adults [4].

Reported scar quality 2 years post burn could be predicted by one patient-related and one injury/ treatment-related characteristic: a dark skin type and more than one operation in the same wound were independently related to lower long-term scar quality, 2 years after the injury [7]. Self-reported scar quality 5 years post burn was predicted by length of hospital stay. In addition, the female gender predicted the overall scar opinion. Thus, all other patient-, injury- or treatment-related potential risk factor predictors did no longer predict scar quality, after taking these predictors into account [8].

Some general remarks can be made based on these findings. Firstly, injury- and treatment-related characteristics are the main predictors of scar outcomes after burn injury. These characteristics are related to burn size (total body surface area burned) and burn depth (number or type of surgery) or the overall healing process in general (length of stay, wound healing complications). Intrinsic patient-related risk factors seem to play a role as well but are less consistent predictors of scar outcome. This includes the risk factors the female gender and also a younger age and darker skin.

An important limitation of three of the aforementioned studies is the sample size. In sample sizes up to 251 patients, only a limited number of predictors can be revealed in multivariate analysis. This was illustrated by the results of the adult study from Wallace et al.; their analyses on 616 people after burns revealed seven significant predictors $[4,10]$, compared to the maximum of three predictors in the other smaller-sized studies.

\subsection{Clinical Relevance}

Knowledge on risk factors for poor scar outcome can be used to tailor treatment, aftercare and scar prevention to these patients with a high-risk profile.

Current evidence shows that injury- and treatmentrelated characteristics are the main predictors of scar outcomes after burn injury. These characteristics are related to burn size (\%TBSA) and burn depth (number or type of surgery) or the overall healing process in general (length of stay, wound healing complications). Intrinsic patient-related risk factors seem to play a role as well but are less consistent predictors of scar outcome. This includes the risk factors the female gender and also a younger age and darker skin.

Future studies, with greater sample sizes, will probably reveal additional predictors for scar outcome. Especially, paediatric scar outcome is not yet well studied.

\subsection{Conclusion}

Early studies on pathological scarring in burn wounds have distinguished between hypertrophic scars and contractures. Prevalences of hypertrophic scarring after burn injuries are reported between $8 \%$ and $67 \%$; a recent prospective study revealed a prevalence of $8 \%$. Data on prevalence of burn scar contractures are limited; reported prevalence at discharge varied between 38 and $54 \%$ and decreased with an increasing time post burn. Prevalence of reconstructive surgery after burn varied between 5 and $20 \%$, up to 10 years post injury.

Factors predicting pathological scar formation after burn injuries include patient, injury and treatment characteristics. Injury- and treatment-related characteristics are the main predictors of scar outcomes after burn injury. These characteristics are related to burn size (total body surface area burned) and burn depth (number or type of surgery) or the overall healing process in general (length of stay, wound healing complications). Intrinsic patient-related risk factors seem to play a role 
as well but are less consistent predictors of scar outcome. This includes the risk factors the female gender and also a younger age and darker skin.

Knowledge on risk factors for poor scar outcome can be used to tailor treatment, aftercare and scar prevention to these patients with a high-risk profile.

\section{Take-Home Messages}

- Pathological scarring in burn wounds can result in hypertrophic scars and/or contractures.

- Reported prevalence of hypertrophic scarring after burn injuries varied between $8 \%$ and $67 \%$.

- Reported prevalence of burn scar contractures varied between 38 and $54 \%$.

- About 5 to $20 \%$ of patient after burn injuries receive reconstructive surgery, reflecting scar pathology.

- Factors predicting pathological scar formation after burn injuries include patient, injury and treatment characteristics.

- Injury- and treatment-related characteristics are the main predictors of scar outcomes after burn injury, including burn size (TBSA burned), burn depth (number of type of surgery) or the overall healing process in general (length of stay, wound healing complications).

- Intrinsic patient-related risk factors, including the female gender and also a younger age and darker skin, are less consistent predictors of scar outcome.

- Knowledge on risk factors for poor scar outcome should be used to tailor treatment, aftercare and scar prevention to these patients with a high-risk profile.

\section{References}

1. Gangemi EN, Gregori D, Berchialla P, et al. Epidemiology and risk factors for pathologic scarring after burn wounds. Arch Facial Plast Surg. 2008;10:93-102. https://doi.org/10.1001/archfaci.10.2.93.

2. van der Wal MB, Verhaegen PD, Middelkoop E, et al. A clinimetric overview of scar assessment scales. J Burn Care Res: Official Publication of the American Burn Association. 2012;33:e79-87. BCR.0b013e318239f5dd.

3. Bombaro KM, Engrav LH, Carrougher GJ, et al. What is the prevalence of hypertrophic scarring following burns? Burns. 2003;29:299-302. DOI: S0305417903000676 [pii].

4. Wallace HJ, Fear MW, Crowe MM, et al. Identification of factors predicting scar outcome after burn in adults: a prospective case-control study. Burns. 2017;43:1271-83.

5. Oosterwijk AM, Mouton LJ, Schouten H, et al. Prevalence of scar contractures after burn: a systematic review. Burns. 2017:43:41-9.

6. Schouten HJ, Nieuwenhuis MK, van Baar M E, et al. The prevalence and development of burn scar contractures. A prospective multicentre cohort study. Submitted.

7. Goei $\mathrm{H}$, van der Vlies $\mathrm{CH}, \mathrm{Hop} \mathrm{MJ}$, et al. Long-term scar quality in burns with three distinct healing potentials: a multicenter prospective cohort study. Wound Repair Regen. 2016;24:721-30.

8. Spronk I, Polinder S, Haagsma J, et al. Patient-reported scar quality of adults after burn injuries: a five-year multicenter follow-up study. Wound Repair Regen Acc.

9. Hop MJ, Langenberg LC, Hiddingh J, et al. Reconstructive surgery after burns: a 10-year follow-up study. Burns. 2014;40:154451. https://doi.org/10.1016/j.burns.2014.04.014.

10. Wallace HJ, Fear MW, Crowe MM, et al. Identification of factors predicting scar outcome after burn injury in children: a prospective case-control study. Burns Trauma. 2017;5:19.

\section{Further Reading}

Finnerty CC, Jeschke MG, Branski LK, Barret JP, Dziewulski P, Herndon DN. Hypertrophic scarring: the greatest unmet challenge after burn injury. Lancet. 2016;388:1427-36.

Open Access This chapter is licensed under the terms of the Creative Commons Attribution 4.0 International License (http://creativecommons. $\mathrm{org} /$ licenses/by/4.0/), which permits use, sharing, adaptation, distribution and reproduction in any medium or format, as long as you give appropriate credit to the original author(s) and the source, provide a link to the Creative Commons license and indicate if changes were made.

The images or other third party material in this chapter are included in the chapter's Creative Commons license, unless indicated otherwise in a credit line to the material. If material is not included in the chapter's Creative Commons license and your intended use is not permitted by statutory regulation or exceeds the permitted use, you will need to obtain permission directly from the copyright holder.

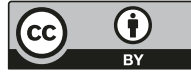

